\title{
The Effect of Chemical Reactivity on the Formation of Gaseous Oblique Detonation Waves
}

\author{
Chian Yan ${ }^{1}$, Hong Hui Teng ${ }^{2}$, Xiao Cheng Mi ${ }^{3}$ and Hoi Dick Ng $^{1, *(\mathbb{C}}$ \\ 1 Department of Mechanical, Industrial and Aerospace Engineering, Concordia University, Montreal, \\ QC H3G 1M8, Canada; y_chia@encs.concordia.ca \\ 2 School of Aerospace Engineering, Beijing Institute of Technology, Beijing 100081, China; hhteng@bit.edu.cn \\ 3 Department of Physics, Cavendish Laboratory, University of Cambridge, Cambridge CB3 0HE, UK; \\ xcm20@cam.ac.uk \\ * Correspondence: hoing@encs.concordia.ca; Tel.: +1-514-848-2424 (ext. 7121)
}

Received: 24 April 2019; Accepted: 24 May 2019; Published: 28 May 2019

\begin{abstract}
High-fidelity numerical simulations using a Graphics Processing Unit (GPU)-based solver are performed to investigate oblique detonations induced by a two-dimensional, semi-infinite wedge using an idealized model with the reactive Euler equations coupled with one-step Arrhenius or two-step induction-reaction kinetics. The novelty of this work lies in the analysis of chemical reaction sensitivity (characterized by the activation energy $E_{\mathrm{a}}$ and heat release rate constant $k_{\mathrm{R}}$ ) on the two types of oblique detonation formation, namely, the abrupt onset with a multi-wave point and a smooth transition with a curved shock. Scenarios with various inflow Mach number regimes $M_{0}$ and wedge angles $\theta$ are considered. The conditions for these two formation types are described quantitatively by the obtained boundary curves in $M_{0}-E_{\mathrm{a}}$ and $M_{0}-k_{\mathrm{R}}$ spaces. At a low $M_{0}$, the critical $E_{\mathrm{a}, \mathrm{cr}}$ and $k_{R, \mathrm{cr}}$ for the transition are essentially independent of the wedge angle. At a high flow Mach number regime with $M_{0}$ above approximately 9.0, the boundary curves for the three wedge angles deviate substantially from each other. The overdrive effect induced by the wedge becomes the dominant factor on the transition type. In the limit of large $E_{\mathrm{a}}$, the flow in the vicinity of the initiation region exhibits more complex features. The effects of the features on the unstable oblique detonation surface are discussed.
\end{abstract}

Keywords: propulsion; oblique detonation; CFD; supersonic combustion; chemical kinetics

\section{Introduction}

In recent years, high efficiency propulsion systems have been sought for their use in the development of air-breathing hypersonic aircrafts [1,2]. The concept of oblique detonation waves has given rise to the development of Oblique Detonation Wave Engines (ODWE) and Ram Accelerators [3-7]. This class of propulsion systems not only has the advantage of the supersonic combustion ramjet (Scramjet) $[8,9]$, but also achieves a high thermal cycle efficiency through the detonation mode of combustion.

A wealth of experimental and numerical investigations can be found in the literature on the formation of oblique detonation wave (ODW) when a hypersonic blunt projectile is launched into combustible mixtures, e.g., References [10-19]. These studies focus primarily on the conditions required to initiate an oblique detonation wave in the combustible mixture. Depending on the test conditions such as the projectile speed and mixture initial conditions, different combustion regimes were observed around the blunt body, e.g., a prompt or delayed ODW, combustion instabilities, a wave splitting, or an inert shock wave. Criteria based on the energetic and kinetic limits are proposed to predict 
the conditions and interpret the observed flow field regimes in terms of competing reactions and flow-quenching effects.

Alternatively, an oblique detonation wave can be induced by a wedge in an incoming reactive flow [20-22]. A standing oblique detonation wave attached to the wedge tip presents a more practical configuration for engine operation [23-25]. There has been indeed a remarkable progress in understanding the fundamental aspects of oblique detonation waves induced by a semi-infinite, two-dimensional wedge. Analytical solutions such as wave angles and steady structures as the basic foundation were sought in a number of pioneering works using detonation polar analysis by approximating the ODW as an oblique shock wave (OSW) coupled with an instantaneous post-shock heat release [26-29]. In later studies, it has been demonstrated that the ODW formation structure induced by the wedge is more complex. In many cases, an oblique shock wave first forms upon the flow interaction with the wedge igniting the combustible flow mixture, and subsequently transits into an oblique detonation wave [30]. Due to the strong coupling sensitivity between fluid dynamics and chemical reactions, as well as the inherent unstable nature of detonation waves [31], it remains technically challenging to establish steady oblique detonations in high-speed combustible mixtures for practical propulsion applications, and such success requires fundamental understanding of the initiation structure of oblique detonation waves. To this end, the dynamics of ODW formation has recently drawn significant research attention.

With the advance in scientific computing using parallel Central Processing Units (CPUs) and Graphics Processing Units (GPUs) computing technology [32-34], there has been significant advance in investigating in detail the unsteady oblique detonation waves. Using high-resolution numerical simulations, various formation structures of wedge-induced oblique detonation waves, i.e., the transition from the oblique shock wave (OSW) to the oblique detonation wave (ODW), have been revealed in recent investigations, e.g., References [35-40]. Hysteresis phenomenon of the ODW structure related to initial condition and ODW responses subject to inflow non-uniformities and turbulences have also been investigated [41-45]. Fundamentally, there exists two key transition types, namely, the abrupt transition from OSW to ODW where a nonreactive oblique shock, a set of deflagration waves, and the oblique detonation surface, all united on a multi-wave point; and the smooth transition characterized by a smoothly curved shock [35,36,46,47], see Figure 1 . It is well established that both the flow conditions and chemical properties of the combustible mixture determine the possible transition type. While it is qualitatively found that the smooth transition usually appears in the cases with a high Mach number and weak chemical sensitivity with a low activation energy of the reactive mixture, the quantitative conditions are yet to be fully determined.

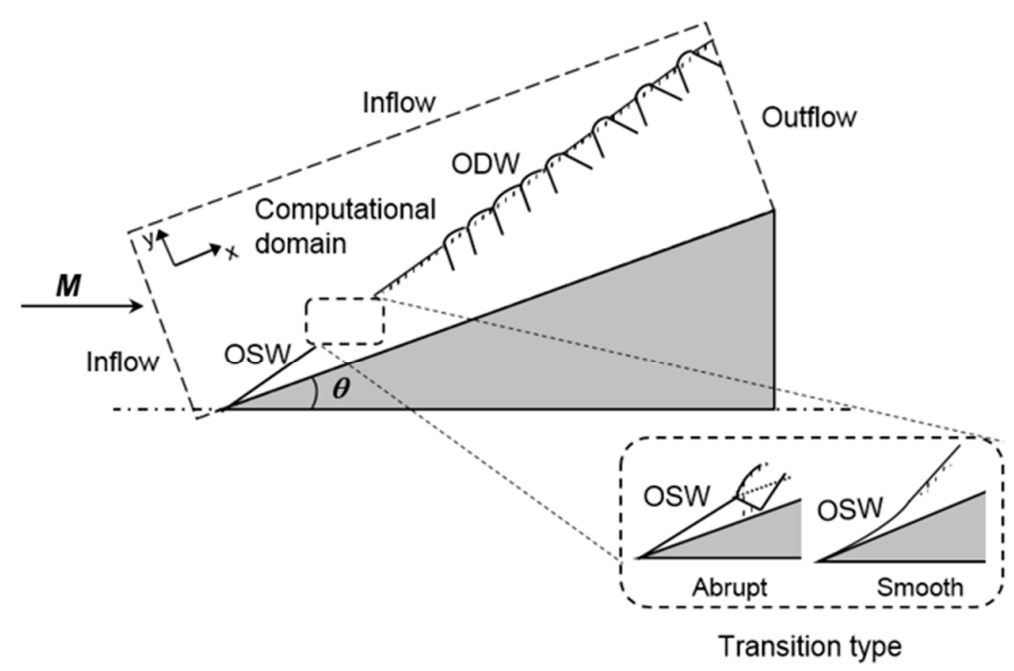

Figure 1. A schematic of the computational details and oblique detonation wave (ODW) phenomena. OSW: oblique shock wave. 
In this work, a parametric numerical study is presented to address the effect of chemical reaction sensitivity and length scales on the ODW initiation structure. Following previous studies, the ODW phenomena are simulated using an ideal-gas, reactive flow model given by the inviscid Euler equations with one-step irreversible Arrhenius or two-step induction-reaction kinetics [48-51]. While numerical simulations using complex chemistry with detailed chemical reaction rates are nowadays possible with increasing computational resources, it remains challenging to fully synthesize and explain the tremendous amount of chemical kinetic and flow field information that are generated from the computation. In fact, although recent studies have shown that detailed chemical kinetics plays an important role in detonation dynamics and its use may introduce additional transient and multiscale effects [52-55], qualitative comparison with simulations using a detailed reaction mechanism has shown the simplified chemistry models, e.g., one-step or two-step chain-branching type kinetics, correctly elucidate the underlying physics of the ODW dynamics, importantly the two transition processes this study is focusing on, e.g., References [38,39,56]. In addition, the two-step induction-reaction kinetics, consisting of a thermally neutral induction step followed by a main heat release reaction layer, provides a compromise on the detailed chemistry model. It retains the simplicity of global kinetics but is detailed enough to mimic salient features of real combustion governed by chain-branching kinetics, allowing the introduction of two length scales, i.e., induction and reaction lengths, apart from the temperature sensitivities governed by the activation energy [48]. In recent studies, it becomes increasing clear that detonation propagation dynamics and initiation are preliminary affected by the induction and chain-branching reaction times or length scales, in addition to the overall reaction sensitivity of the combustible mixture and its energetics [57,58]. All these chemical kinetic characteristics can be elucidated by the two simplified reaction models considered in this work. It is thus natural to carry simulations to first observe quantitatively the ODW formation under the influence of these global kinetic parameters.

The use of the simplified kinetic models is thus a preliminary step in understanding the phenomena and deriving interpretable quantitative data. It is worth mentioning again that with the use of realistic detailed chemistry, besides being capable to interpret a large amount of computational data, numerical issues such as accumulation of errors and resolving added multiscale reactive dynamics become increasingly important. The latter complexity in the chemical kinetics requires extremely high numerical resolution for the reliable simulations. To this end, interpretation of the CFD data returns to developing reduced models in order to simplify the understanding of the flow phenomena. Hence, simulations with reduced kinetics are again unavoidable for comparison.

In this parametric study, computations were carried out over a wide range of activation energy $E_{\mathrm{a}}$ (non-dimensionalized with respect to the initial pre-shock state) from 20 to 60 using the one-step Arrhenius kinetics, heat release rate constant $k_{\mathrm{R}}$ from 1 to 10 for the two-step kinetics, three wedge angles $\theta$ and different inflow Mach number $M_{0}$. It is worth noting that the high $E_{\mathrm{a}}$ regime considered in this work has not been explored thoroughly in any previous studies due to the numerical resolution requirement. Boundaries separating the two aforementioned transition types are obtained and new features resulting from the effect of high activation energy on the flow structure in the vicinity of the ODW initiation region and the fully developed ODW unstable surface are discussed in detail.

\section{Computational Methods}

The schematic shown in Figure 1 describes also the computational setup. The computational domain bounded by the dashed lines is rotated to the direction along the wedge surface. The Cartesian grid in this rectangular domain is thus aligned with the wedge surface and the inflow velocities to the computational domain are determined and projected based on the rotation angle. Inflow conditions are thus employed for the left and top boundaries; outflow conditions extrapolated from the interior are implemented on the right boundary and few grid cells before the wedge tip. Slip reflective boundary condition is used on the wedge surface. 
Qualitatively, a supersonic flow with Mach number $M_{0}$ enters the domain from the left and is reflected on the two-dimensional wedge with an angle $\theta$. An OSW is generated and triggers the chemical reaction. After an induction period, the transition to an oblique detonation occurs. Neglecting the viscous and diffusive effects, which are shown to have insignificant influence on the overall ODW structure [59], the governing equations for the ODW flow dynamics are simplified into the reactive Euler equations as follows:

$$
\begin{aligned}
& \frac{\partial \mathbf{U}}{\partial t}+\frac{\partial \mathbf{F}(\mathbf{U})}{\partial x}+\frac{\partial \mathbf{G}(\mathbf{U})}{\partial y}=\mathbf{S}(\mathbf{U}) \\
& \mathbf{U}=\left(\begin{array}{c}
\rho \\
\rho u \\
\rho v \\
\rho e \\
\rho \lambda
\end{array}\right), \quad \mathbf{F}(\mathbf{U})=\left(\begin{array}{c}
\rho u \\
\rho u^{2}+p \\
\rho u v \\
(\rho e+p) u \\
\rho u \lambda
\end{array}\right), \quad \mathbf{G}(\mathbf{U})=\left(\begin{array}{c}
\rho v \\
\rho u v \\
\rho v^{2}+p \\
(\rho e+p) v \\
\rho v \lambda
\end{array}\right), \mathbf{S}(\mathbf{U})=\left(\begin{array}{c}
0 \\
0 \\
0 \\
0 \\
\rho \dot{\omega}
\end{array}\right)
\end{aligned}
$$

where $e$ is the total energy per unit mass given by:

$$
e=\frac{p}{(\gamma-1) \rho}+\frac{\left(u^{2}+v^{2}\right)}{2}+\lambda Q
$$

All the flow variables and chemical parameters have been made dimensionless by reference to the uniform unburned state ahead of the detonation front, i.e.,

$$
\rho=\frac{\widetilde{\rho}}{\rho_{0}}, p=\frac{\widetilde{p}}{p_{0}}, T=\frac{\widetilde{T}}{T_{0}}, u=\frac{\widetilde{u}}{\sqrt{R T_{0}}}, x=\frac{\widetilde{x}}{x_{\mathrm{ref}}}, Q=\frac{\widetilde{Q}}{R T_{0}}, E_{a}=\frac{\widetilde{E_{a}}}{R T_{0}}
$$

The above governing equations are coupled with a chemical kinetic law for the reaction rate $\dot{\omega}$. In this study, two different reaction rate laws were considered, i.e., single-step kinetics and two-step induction-reaction kinetics. The single-step irreversible chemical reaction model with an Arrhenius rate law is given as:

$$
\dot{\omega}=-k \lambda \exp \left(\frac{-E_{a}}{T}\right)
$$

in which the reaction sensitivity is governed by $E_{\mathrm{a}}$. The pre-exponential constant for a given mixture $k$ is used to define the spatial and temporal scales, so by solving the steady Zel'dovich-von Neumann-Döring (ZND), one-dimensional, Chapman-Jouguet (CJ) detonation, the half reaction zone length $l_{1 / 2}$ is unity $[60,61]$. Following previous studies, [62-66], the normalized heat release $Q$ is chosen to be 50 and the isentropic exponent $\gamma=1$.2. For the activation energy range considered in this work, i.e., $E_{\mathrm{a}}=5$ to $60, k$ varies from 1.8 to 14,640 . Mixture parameters and corresponding ZND Chapman-Jouguet (CJ) detonation properties are provided in Table 1.

Table 1. Mixture parameters and corresponding Chapman-Jouguet (CJ) detonation properties.

\begin{tabular}{cc}
\hline Parameters & Values \\
\hline Heat release, $Q$ & 50 \\
Ratio of specific heats, $\gamma$ & 1.2 \\
Post-shock temperature, $T_{\mathrm{S}}$ & 4.814 \\
Post-shock temperature, $P_{\mathrm{s}}$ & 42.063 \\
Post-shock particle velocity, $u_{\mathrm{vn}}$ & 0.7792 \\
CJ detonation temperature, $T_{\mathrm{CJ}}$ & 11.998 \\
CJ detonation pressure, $P_{\mathrm{CJ}}$ & 21.531 \\
CJ detonation Mach number, $M_{\mathrm{CJ}}$ & 6.2162 \\
\hline
\end{tabular}


A more realistic reaction model includes an induction zone [48-51] with an additional advection equation for $\xi$. The reaction zone is modeled in the same way and the induction zone is also of an Arrhenius type as follows:

$$
\begin{gathered}
\dot{\omega}_{I}=H(1-\xi) \cdot k_{I} \exp \left[E_{I}\left(\frac{1}{T_{S}}-\frac{1}{T}\right)\right] \\
\dot{\omega}_{R}=-(1-H(1-\xi)) \cdot \lambda k_{R} \exp \left(\frac{-E_{R}}{T}\right)
\end{gathered}
$$

where $\xi$ is the progress variable in the induction process and $H(1-\xi)$ is a step function, i.e.,:

$$
H(1-\xi)\left\{\begin{array}{l}
=1 \text { if } \xi<1 \\
=0 \text { if } \xi \geq 1
\end{array}\right.
$$

In the two-step kinetic model, the reference length scale $x_{\text {ref }}$ is chosen such that the one-dimensional ZND induction length $\Delta_{\mathrm{I}}$ is unity, i.e., $k_{\mathrm{I}}=-u_{\mathrm{vn}}$ where $u_{\mathrm{vn}}$ is the particle velocity behind the shock front in the shock-fixed frame for the Chapman-Jouguet (CJ) detonation. $T_{\mathrm{s}}$ is the temperature at the shocked state of the ZND detonation. Again, the local chemical energy that has been released at any instant during the reaction is equal to $\lambda Q$ where $Q$ is also set to 50 . There are two associated activation energies, namely $E_{\mathrm{I}}$ and $E_{\mathrm{R}}$, which are rescaled by the temperature jump across the leading shock of the detonation to give $\varepsilon_{\mathrm{I}}=5.0 T_{\mathrm{s}}$ and $\varepsilon_{\mathrm{R}}=1.0 T_{\mathrm{s}}$. The pre-exponential factor $k_{\mathrm{R}}$ is used in this work to vary the reaction zone structure [48]. The solutions to the above equation systems are obtained numerically using a 2nd order MUSCL-Hancock scheme with an HLLC Riemann solver [67,68], with a CFL number of 0.90 . To reduce the simulation run-time, the entire flow solver was implemented using NVIDIA CUDA programming language (NVIDIA Corp., Santa Clara, CA, USA) and run on a NVIDIA Tesla K40 General Purpose Graphics Processing Unit GPGPU [68-70]. Application of the GPU-CPU framework improves significantly the computational performance allowing high resolution simulations and parametric study to be performed efficiently.

To capture the complete ODW formation structure and to adjust the computational cost, different computational domains are used, i.e., $80 \times 30,160 \times 60$ or $220 \times 80$ for the one-step kinetics and $120 \times 40,150 \times 50$ for the two-step kinetic model, depending on the initial flow and mixture conditions. Three wedge angles $\theta$ are considered in this study, i.e., $26^{\circ}, 28^{\circ}$, and $30^{\circ}$.

\section{Results and Discussion}

An important numerical issue that requires attention is that, in most of the former studies, a numerical resolution of 32 pts (or less) per characteristic reaction zone length of a CJ-ZND detonation ( $l_{1 / 2}$ and $\Delta_{\mathrm{I}}$ for single- and two-step kinetic model, respectively) is used in computations. However, when activation energy reaches a higher value or the heat release rate becomes stiff, a much higher numerical resolution is required to achieve numerical convergence $[62,63,65]$. Figure 2 shows the different temperature contours for the case of $M_{0}=12.5, \theta=26^{\circ}$ and high $E_{\mathrm{a}}=60$ for the one-step Arrhenius model with increasing grid resolutions from 32,64 to 128 pts per half reaction zone length. It is clear that, from Figure 2, for $E_{\mathrm{a}}=60,32$ grid points per half reaction zone length is insufficient. The fore part of the unstable ODW structure and the Kelvin-Helmholtz $(\mathrm{K}-\mathrm{H})$ vortex-rolling along the shear layer cannot be revealed. Only when the resolution is increased to $64 \mathrm{pts}$, the global features (e.g., initiation point, onset of cellular ODW surface instability, K-H instability, etc.) converge as those compared well with the results obtained using 128 pts. Taking this issue into account, the default resolution considered in this work is 64 pts per half reaction zone length for $E_{\mathrm{a}}$ less than 30 and only for higher $E_{\mathrm{a}}$ values a grid resolution of $128 \mathrm{pts} / l_{1 / 2}$ is used. Equivalently, a resolution study result is also provided in Figure 3 for the two-step induction-reaction kinetics for the case of $M_{0}=10.0, \theta=30^{\circ}$, and $k_{\mathrm{R}}=2.8$. For a more quantitative comparison, the pressure and temperature profiles along the line $y=5$ are shown in Figure 4. Overall, the curves are overlapped together so the effects of different grids 
are indeed found negligible. These results confirm again that a resolution of 64 pts per ZND induction zone length is sufficient.

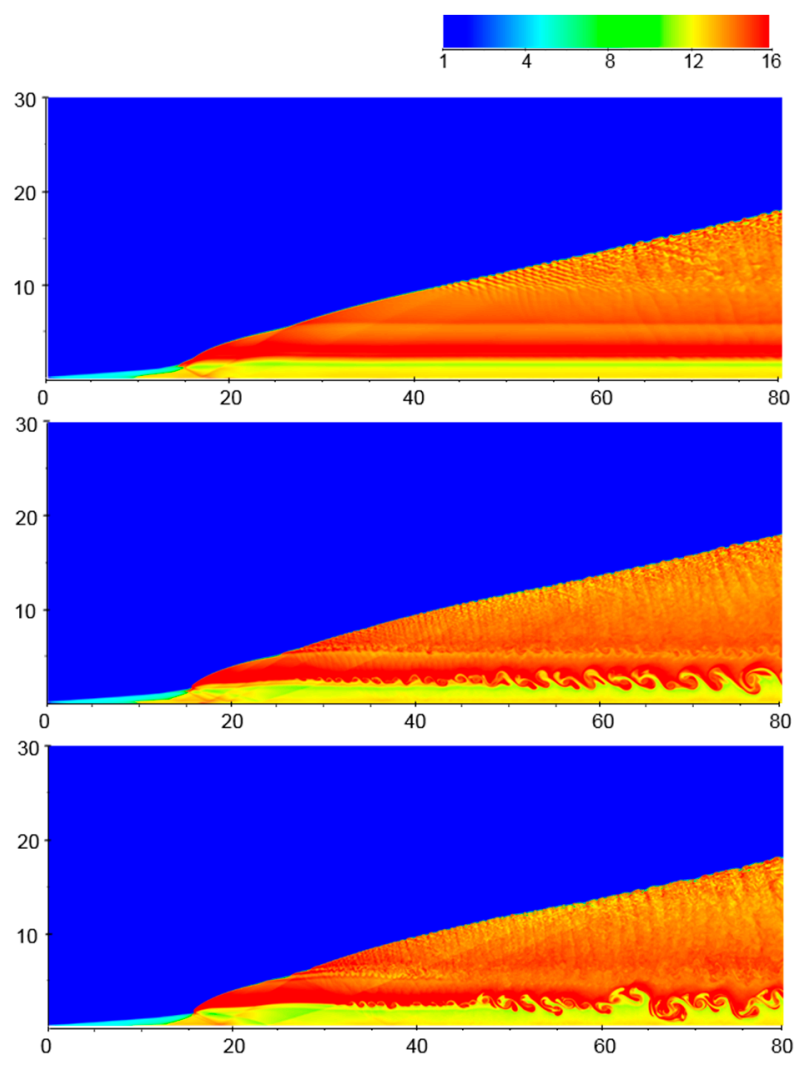

Figure 2. Temperature contours with 32 (upper), 64 (middle) and 128 (lower) pts $/ l_{1 / 2}$ for $M_{0}=12.5$, $\theta=26^{\circ}$ and $E_{\mathrm{a}}=60$ for the one-step Arrhenius kinetics with $k=14,640$.
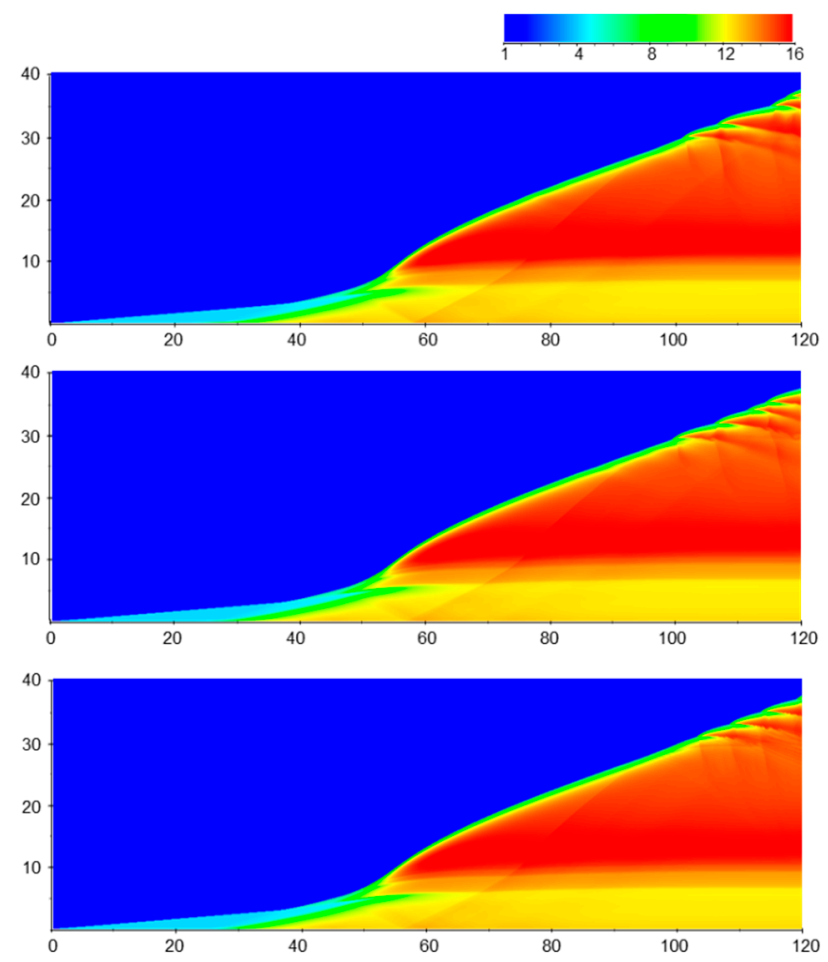

Figure 3. Temperature contours with 32 (upper), 64 (middle) and 128 (lower) pts $/ \Delta_{\mathrm{I}}$ for $M_{0}=10.0$, $\theta=30^{\circ}$ and $k_{\mathrm{R}}=2.8$ for the two-step induction-reaction kinetics. 

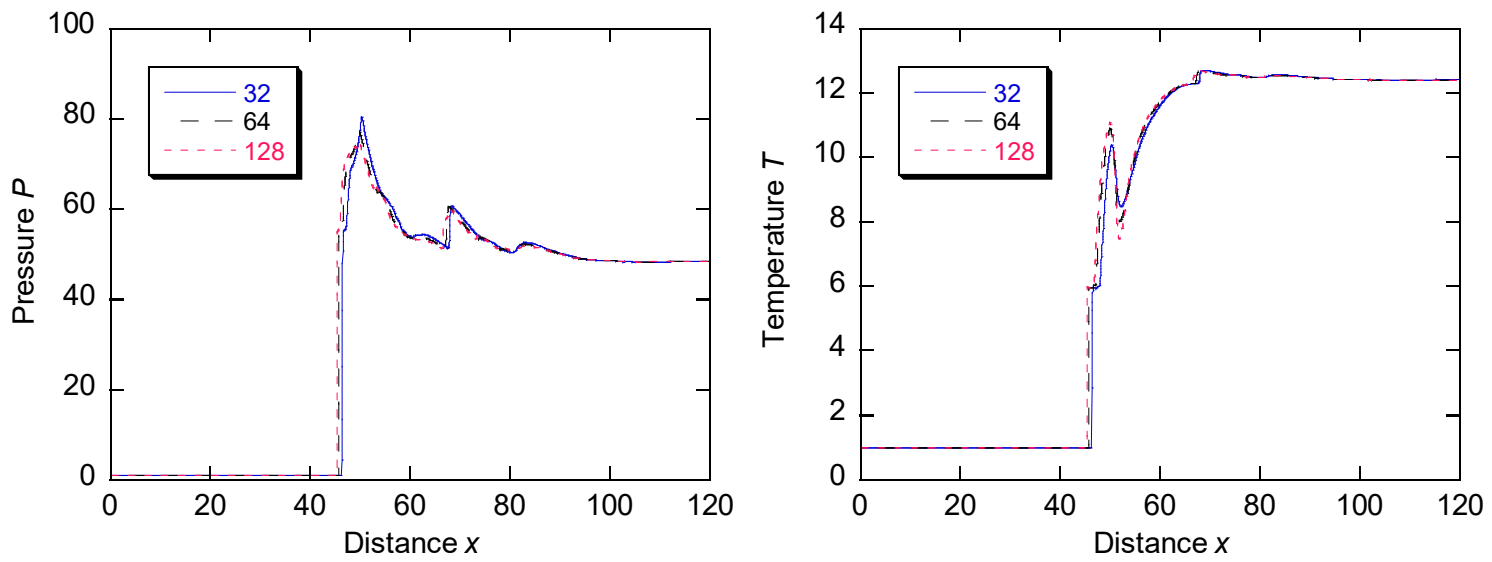

Figure 4. Pressure (left) and temperature (right) profiles along the line $y=05$ obtained using different grid resolutions with $M_{0}=10.0, \theta=30^{\circ}$ and $k_{\mathrm{R}}=2.8$ for the two-step induction-reaction kinetics.

Besides effects of numerical resolution, studies have shown that it is also important to take into consideration the accumulation of errors from each time-integration step for reactive flow simulations [71,72]. In other words, the effect of time-step size needs also to be verified on the reliability of the results. Therefore, simulations with different numbers of time steps by varying the Courant-Friedrichs-Lewy (CFL) number (i.e., reducing the CFL number to 0.50 and 0.25 ) are performed for cases with both one-step Arrhenius kinetics and two-steps induction-reaction models, see Figures 5 and 6. It can be observed from the comparison, together with Figures 2 and 3, that the overall flow fields such as the wave structure around the initiation region and the location of the onset of ODW are not affected by the CFL number variation. Hence, the default CFL number of 0.90 was applied for all the following simulations.
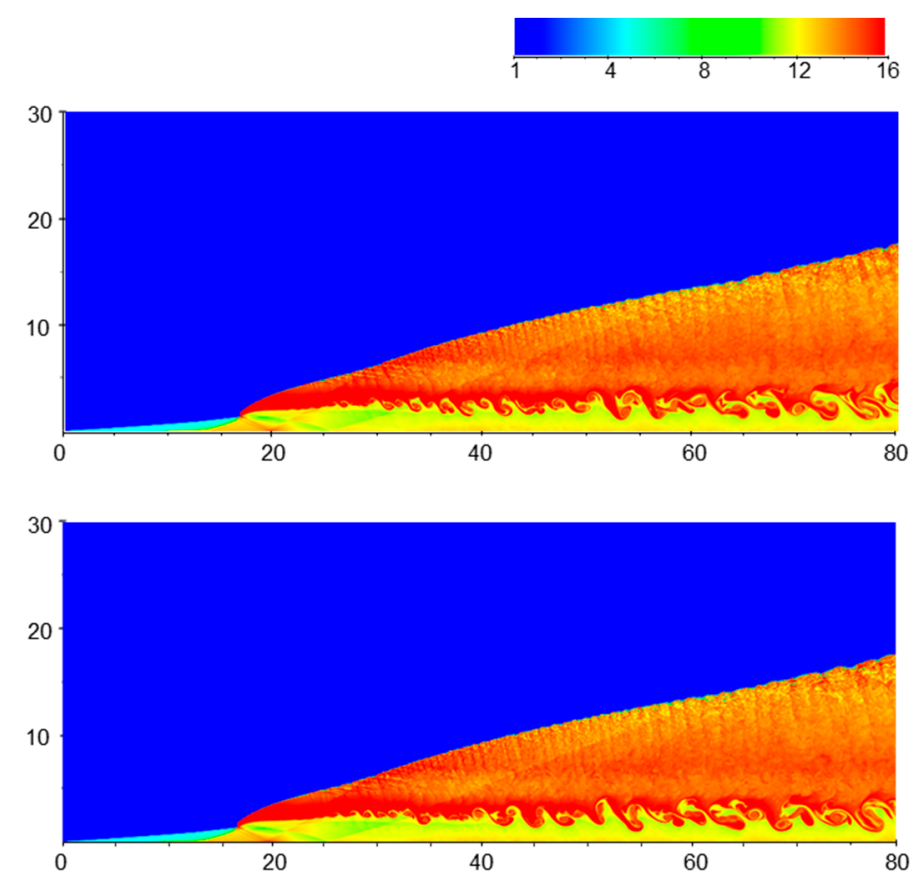

Figure 5. Temperature contours for the case $M_{0}=12.5, \theta=26^{\circ}$ and $E_{\mathrm{a}}=60$ obtained using the one-step Arrhenius kinetics with $k=14,640$ and Courant-Friedrichs-Lewy (CFL) number equal to 0.5 (upper); and 0.25 (lower). 

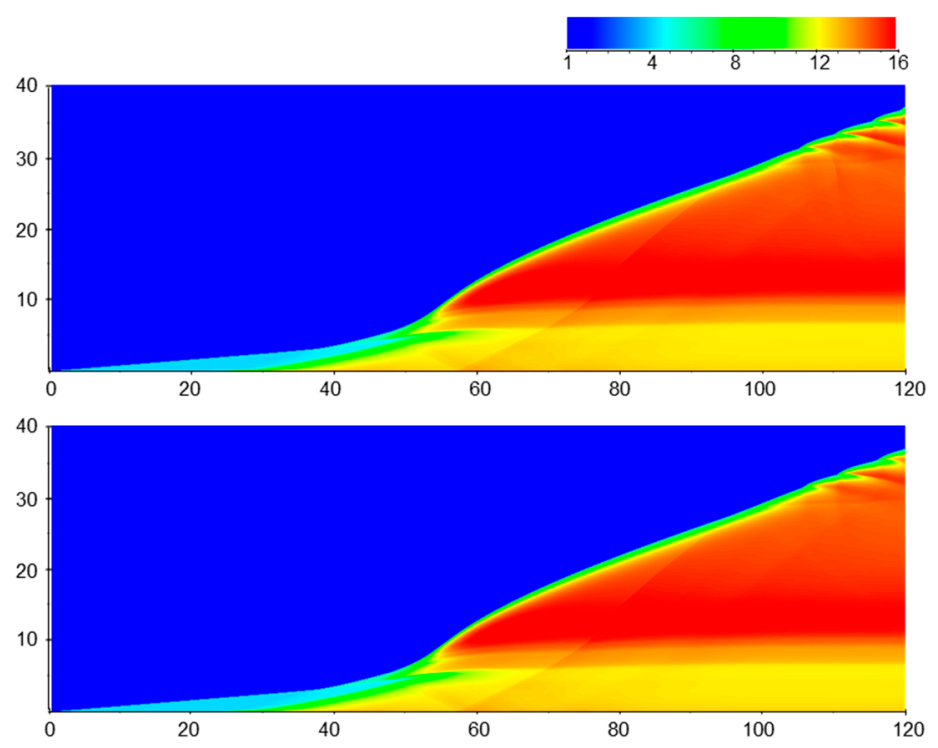

Figure 6. Temperature contours for the case $M_{0}=10.0, \theta=30^{\circ}$ and $k_{\mathrm{R}}=2.8$ using the two-step induction-reaction kinetics and CFL number equal to 0.5 (upper); and 0.25 (lower).

Figure 7 first shows the ODW formation structures obtained with $\theta=26^{\circ}, M_{0}=12.5$ and 10, and $E_{\mathrm{a}}=35$ for the one-step Arrhenius kinetic model. Consistent with the finding in the literature $[36,40,64]$, the transition is characterized by a smoothly curved shock at $M_{0}=12.5$, while at low Mach number, the transition is given by the classical abrupt structure. For the case with $M_{0}=10$, the multi-wave point connecting the deflagration wave, induction OSW, ODW, and transverse compression waves can be clearly seen. A slip line extending downstream from the abrupt point can also be observed. Equivalently, Figure 8 elucidates the two types of transition with a fixed inflow $M_{0}=10$ and $\theta=26^{\circ}$ but with various activation energies $E_{\mathrm{a}}$ from 20 to 30. Clearly, these results demonstrate that not only the inflow condition controls the transition process, the chemical sensitivity is another dominant parameter on the initiation evolution. The smooth transition can only be achieved by a mixture with very low temperature sensitivity (or equivalently a small $E_{\mathrm{a}}$ ).
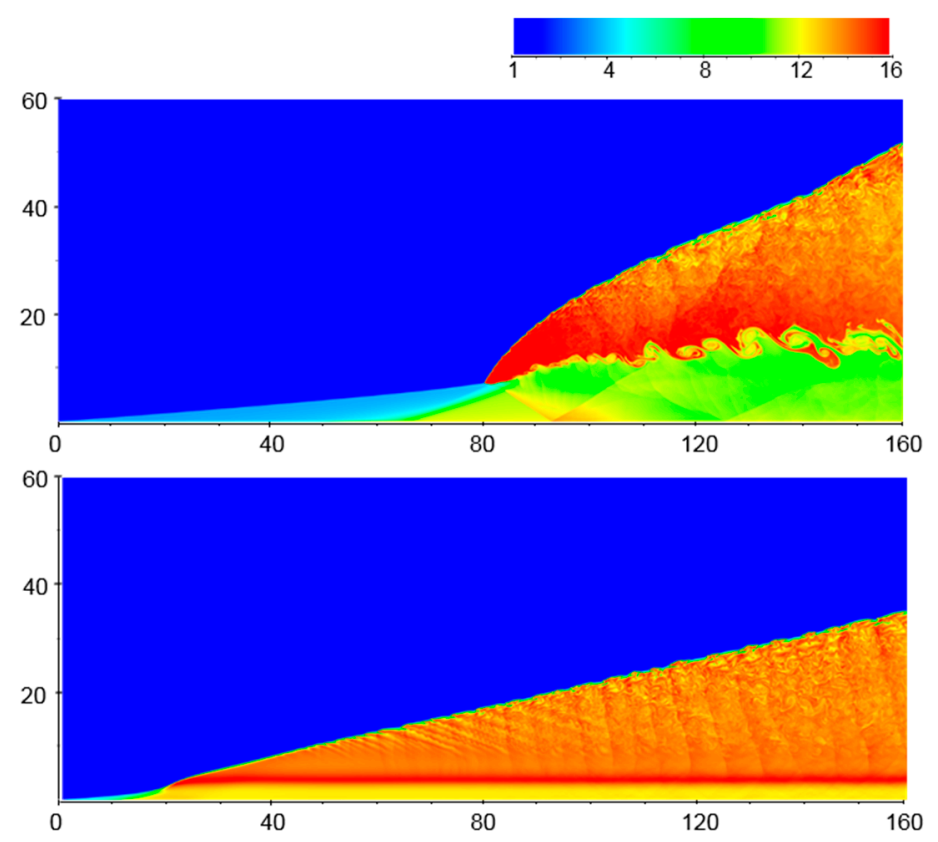

Figure 7. Temperature contours with $E_{\mathrm{a}}=35, k=185.0$ and $\theta=26^{\circ}$ for $M_{0}=10$ (upper) and $M_{0}=12.5$ (lower). 

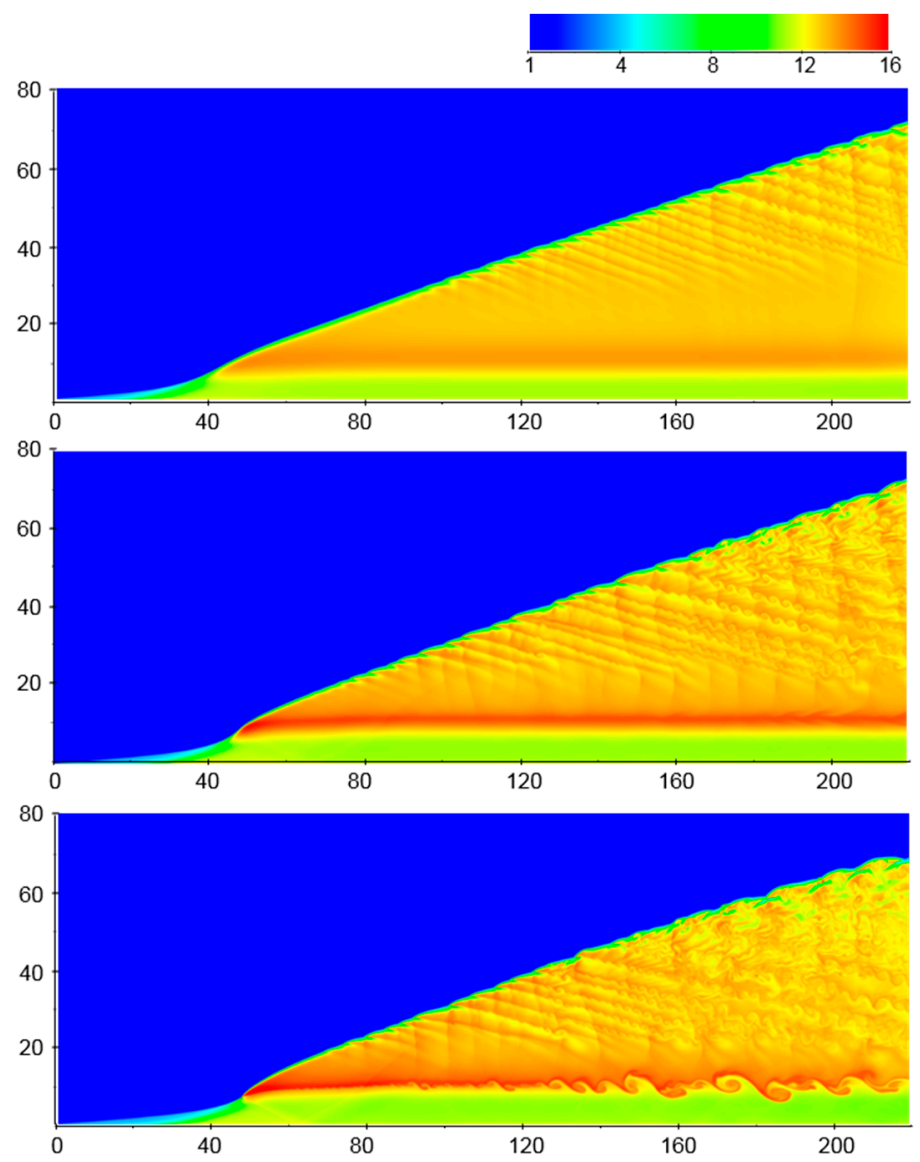

Figure 8. Temperature contours with $M_{0}=10$ and $\theta=26^{\circ}$ for $E_{\mathrm{a}}=20$ (upper) 24 (middle) and 30 (lower).

The corresponding pre-exponential constant $k$ values are 16.6, 31.0, and 81.1, respectively.

Using the one-step Arrhenius kinetic model, the activation energy $E_{\mathrm{a}}$ controls the temperature sensitivity of the reaction rate. To examine the effect of the reaction zone length scale, a parametric study is carried out using the two-step induction-reaction kinetic model. The pre-exponential factor, $k_{R}$, is varied to probe how the ODW formation is affected by the heat release length. Results are presented in Figure 9 for three different $k_{R}$ values. It is also found that, by increasing $k_{R}$, and hence, shortening the heat release length, the resulting ODW formation transits from a smooth to an abrupt configuration. Hence, not only the temperature sensitivity of the reaction zone plays a role in determining the transition type, the heat release length represents another important factor. Similar to Figure 7 , Figure 10 illustrates the effect of inflow Mach number for a mixture governed by induction-reaction kinetics. The two transition types are also observed by changing the Mach number from 9 to 11 with a fixed $k_{\mathrm{R}}=2.5$ and $\theta=30^{\circ}$. 

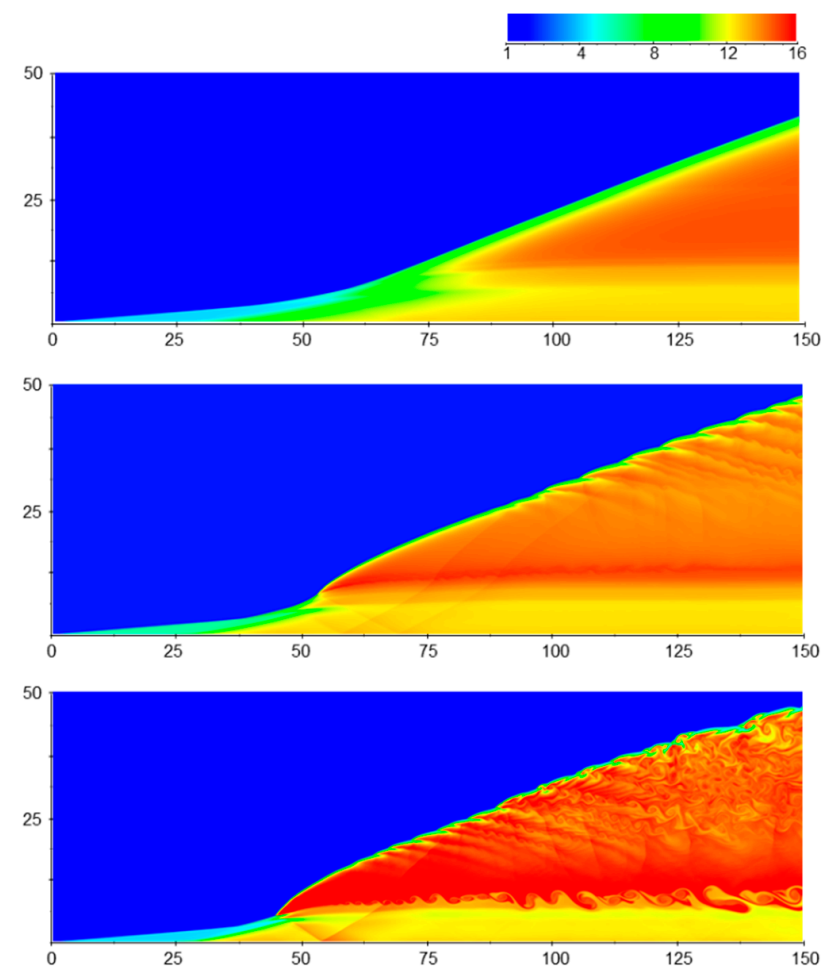

Figure 9. Temperature contours with $M_{0}=10$ and $\theta=30^{\circ}$ for $k_{\mathrm{R}}=1.0$ (upper); 2.98 (middle); and 5.0 (lower).
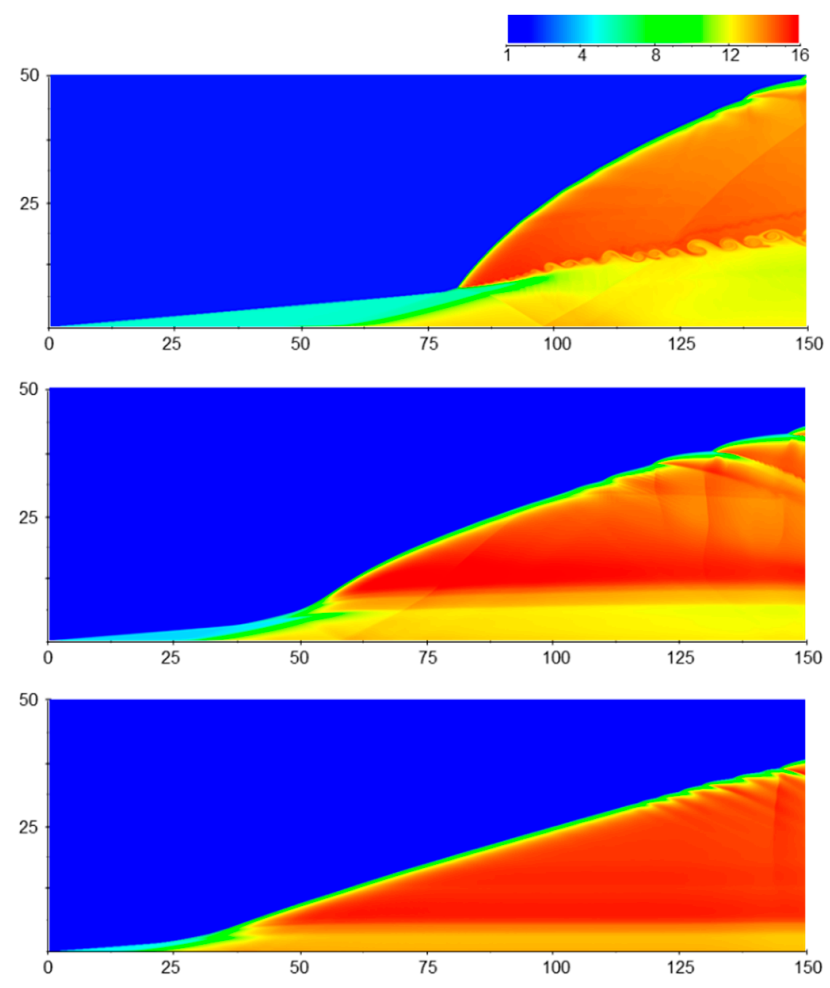

Figure 10. Temperature contours with $k_{\mathrm{R}}=2.5$ and $\theta=30^{\circ}$ for $M_{0}=9.0$ (upper); 10.0 (middle); and 11.0 (lower).

By carrying a full parametric study boundary curves in $E_{\mathrm{a}, \mathrm{cr}}-M_{0}$ and $k_{\mathrm{R}, \mathrm{cr}}-M_{0}$ spaces distinguishing the two types of OSW-ODW transition for different wedge angle $\theta$ are obtained. These curves are 
shown in Figures 11 and 12 for the one-step Arrhenius and two-step induction-reaction kinetic models, respectively.

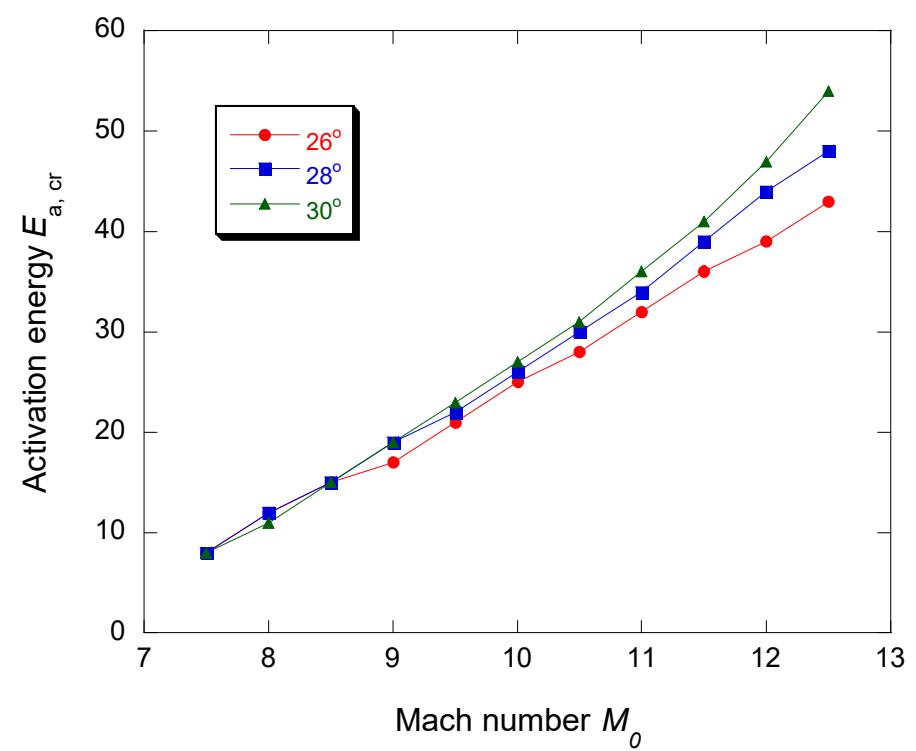

Figure 11. The conditions for smooth and abrupt transitions below and above the boundary respectively in the $E_{\mathrm{a}, \mathrm{cr}}-M_{0}$ plane for three different wedge angles $\theta$.

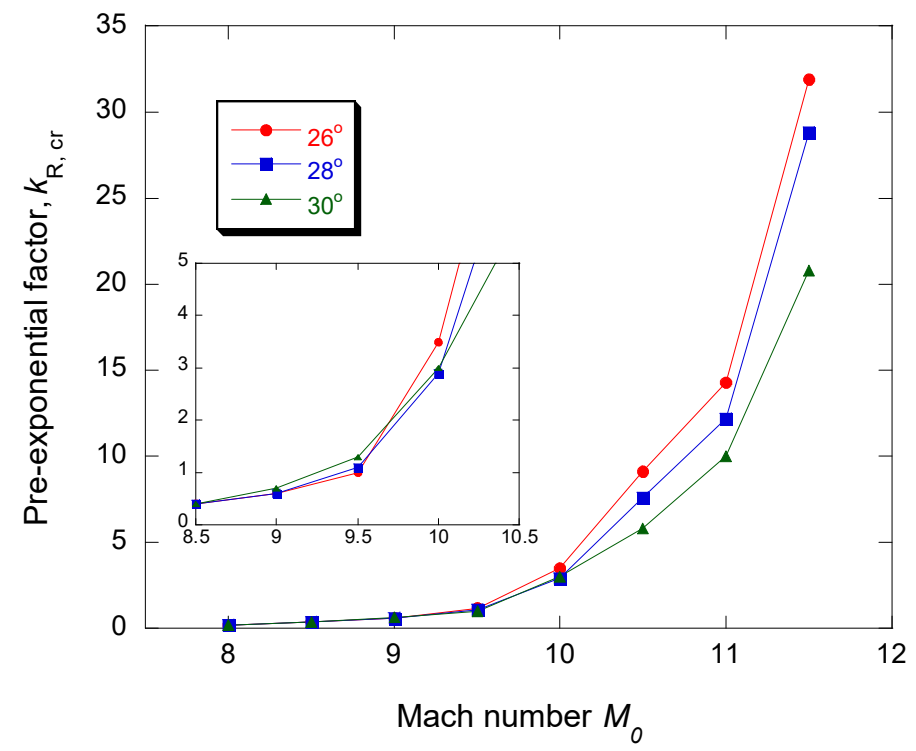

Figure 12. The conditions for smooth and abrupt transitions below and above the boundary respectively in the $k_{\mathrm{R}, \mathrm{cr}}-M_{0}$ plane for three different wedge angles $\theta$.

In Figure 11, it is observed that for all wedge angles $\theta$ a nearly linear relationship in most part between lower $M_{0}$ regime and the corresponding critical transition $E_{\mathrm{a}, \mathrm{cr}}$ is achieved. Only at high $M_{0}$ regime, the curve for the high wedge angle $\theta=30^{\circ}$ begins to deviate prominently. It can be observed from this plot that, at low $M_{0}$ regime, all three curves collapse, i.e., the critical $E_{\mathrm{a}, \mathrm{cr}}$ for the transition is independent of the wedge angle. In other words, the chemical kinetics is thus the controlling factor on the transition pattern of the ODW. As Mach number increases to very high values, the boundary curves for the three-wedge angles deviate from each other. This can be explained by the fact that, at high $M_{0}$ regime, the resulting overdrive effect by the presence of the wedge with greater angles $\theta$ becomes the dominant factor on the transition type. 
Similar trend is also observed in Figure 12 showing the boundary in $k_{\mathrm{R}, \mathrm{rr}}-M_{0}$ space. At low Mach number regime, all curves for different angles are close to each other. The transition occurs at small $k_{R}$, i.e., at relatively larger heat release length. As the inflow Mach number increases, $k_{R, c r}$ increases exponentially and again, the three curves start to deviate from each other. This deviation at high Mach number regime and increasing angles can be again explained as a result of the overdriving effect becoming the dominant mechanism underlying the transition type.

In order to quantify the deviation of the results between different wedge angles with increasing Mach number, considering the lowest wedge angle $\theta=26^{\circ}$ as the baseline case the percentage difference of the critical values $E_{\mathrm{a}, \mathrm{cr}}$ and $k_{\mathrm{R}, \mathrm{cr}}$ between it and results with increasing wedge angle, i.e., $\theta=28^{\circ}$ and $30^{\circ}$ are plotted in Figure 13. It appears the deviation begins at about $M_{0}=9.0$ in both cases with one-step Arrhenius kinetics and two-step induction-reaction kinetics. Below this critical Mach number, the boundaries are indeed the same for all wedge angles at the low Mach number regime.
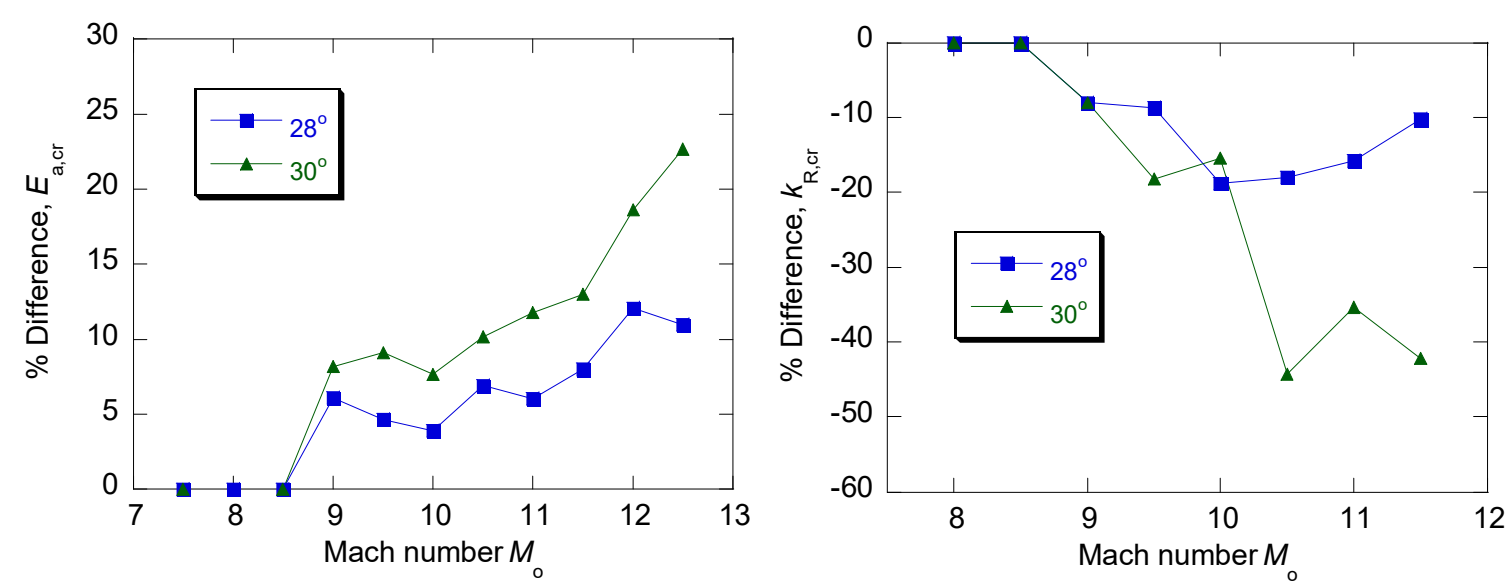

Figure 13. The percentage difference of $E_{\mathrm{a}, \mathrm{cr}}$ (left); and $k_{\mathrm{R}, \mathrm{cr}}$ (right) between the base case of $\theta=26^{\circ}$ and increasing wedge angles $\theta=28^{\circ}$ and $30^{\circ}$.

Some values of the overdrive, determined as $f=\left(M_{0} \cdot \sin \beta / M_{C \mathrm{~J}}\right)^{2}$, where $\beta$ and $M_{\mathrm{CJ}}$ are the oblique detonation angle and Mach number of the $C J$ detonation for a given $Q$ and $\gamma$, respectively, are tabulated in Table 2. In fact, it is known that the heat release length scale has an effect on the instability of detonation, i.e., the shorter the heat release length, the more sensitive the detonation wave to flow perturbation. First, by keeping the same level of temperature sensitivity, i.e., same activation energies for both the induction and reaction steps, overall the higher Mach number and wedge angle have a stabilizing effect by reducing the overall temperature sensitivity of the reaction. Hence, a much greater value of $k_{R}$ thus tends to reduce the heat release length and cause the transition from a smooth to an abrupt formation type. Second, for increasing overdrive, the temperature in the induction region is higher. This, in turn, increases the subsequent heat release rate in the reaction zone and shortens the heat release length. This provides a possible explanation as to why the critical $k_{\mathrm{R}}$ decreases for increasing wedge angle at the high Mach number regime due to the increase of $f$.

Table 2. Degree of overdrive $f$ for different inflow Mach number $M_{0}$ and wedge angle $\theta$.

\begin{tabular}{ccc}
\hline $\boldsymbol{M}_{\mathbf{0}}$ & $\boldsymbol{\theta}$ & $\boldsymbol{f}$ \\
\hline 9 & 26 & 1.20 \\
9 & 28 & 1.27 \\
9 & 30 & 1.36 \\
12 & 26 & 1.50 \\
12 & 28 & 1.63 \\
12 & 30 & 1.77 \\
\hline
\end{tabular}


In a number of previous studies on ODW, the high Mach number inflow regime and a one-step Arrhenius kinetic model with low to moderate $E_{\mathrm{a}}$ are considered. In fact, by further increasing $E_{\mathrm{a}}$ in a high $M_{0}$ regime, the ODW flow field becomes more unstable, thus giving rise to more complex features. Figure 14 shows the results obtained for $\theta=26^{\circ}$ and $M_{0}=12.5$ with a large activation energy $E_{\mathrm{a}}=60$, under which the transition is of an abrupt type. In the limit of large $E_{\mathrm{a}}$, more instabilities begin to appear on the downstream fully-developed ODW surface. The increase of $E_{\mathrm{a}}$ makes the ODW cellular surface more irregular. For large $E_{\mathrm{a}}$, the slip line also becomes unstable and the classical roll-up behavior of the K-H instability in the detonation product becomes more apparent. Similar to normal cellular detonation [73-75], for large $E_{a}$, the ODW cellular surface also becomes highly irregular with unburned pockets behind the leading ODW front [65,76-78].

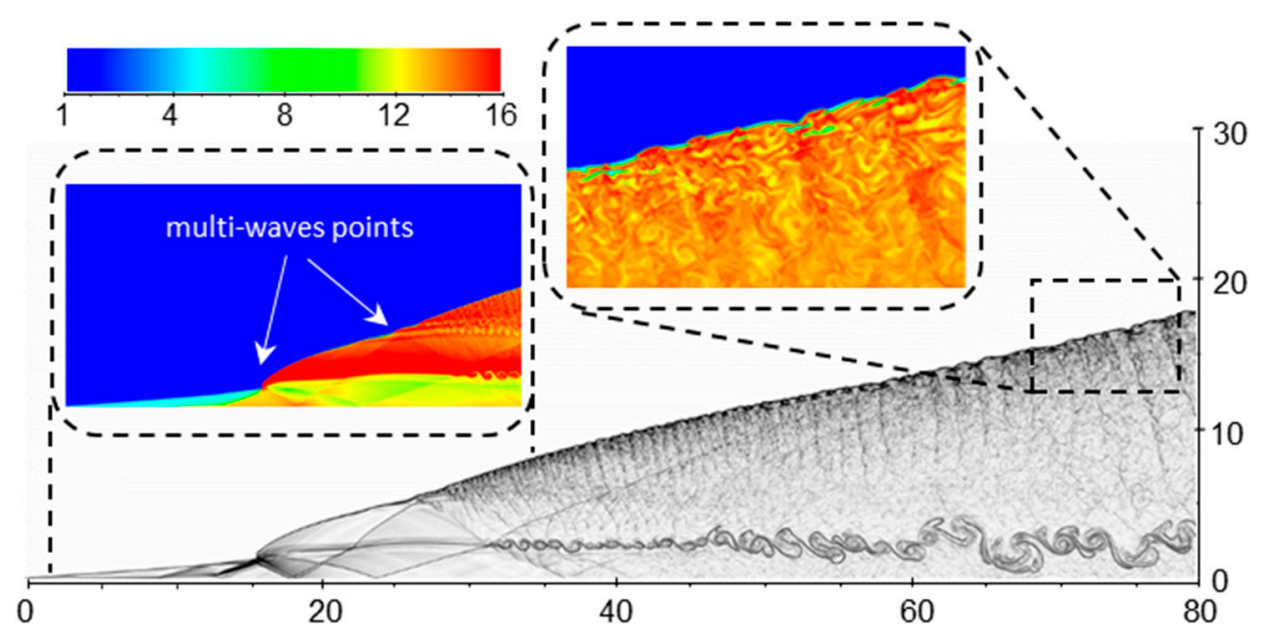

Figure 14. Schlieren plot and temperature contours (sub-plot) with $128 \mathrm{pts} / l_{1 / 2}$ for $M_{0}=12.5, E_{\mathrm{a}}=60$, $k=14,640$ and $\theta=26^{\circ}$.

Another key feature is revealed near the initiation region. It is shown that the transverse compression wave resulting from the initiation point and reflected between the wall and the slip line becomes stronger as $E_{\mathrm{a}}$ increases. The reflected compression wave from the wall further adjusts the gas parameters of the combustion products and penetrates the slip line, and influences the initially smooth ODW surface. The result of this interaction is the formation of another multi-wave point similar to the first initiation point. A second slip line, although faint, can be seen downstream, which eventually becomes unstable far downstream within the combustion product due to the K-H instability. At $E_{\mathrm{a}}=60$, this second multi-wave point appears to be close to the first appearance of transverse waves after another segment of smooth ODW from the main initiation location. For future works, computations taking into account viscous and diffusion effects by solving the Navier-Stokes equations $[79,80]$ need to be considered in order to explore more quantitatively the aforementioned features obtained for large $E_{\mathrm{a}}$ such as the strong $\mathrm{K}-\mathrm{H}$ instability along triple-point shear layers and the unstable oblique detonation cellular structure.

\section{Conclusions}

In summary, this paper investigates the effects of chemical reaction kinetics on ODW initiation using high-resolution numerical simulations. Two simplified kinetic models, namely the conventional one-step Arrhenius kinetics over a wide range of activation energy $E_{\mathrm{a}}$ and the two-step induction-reaction kinetics are considered. The use of a GPU-based solver allows the simulations to be performed using high numerical resolution and a parametric study to be carried out efficiently. The present results obtained using a different computing platform further confirm the validity of the data obtained in previous studies using the conventional CPU computing. For the first time, this study provides quantitative results of the critical conditions distinguishing the two known ODW formation 
types in the $M_{0}-E_{\mathrm{a}}$ and $k_{\mathrm{R}, \mathrm{cr}}-M_{0}$ planes using the one-step Arrhenius and two-step models, respectively. At low Mach number regimes, all critical boundary curves with different wedge angles are close to each other, indicating that the transition is dominated by chemical kinetics. As inflow Mach number increases, the results for different wedge angles start to deviate from each other. This deviation can be explained by the fact that, at the high Mach number regime, the overdrive factor in turn plays a prominent role for determining the transition type. For the one-step Arrhenius case, flow features are revealed in finer details in the limit of large activation energy, including the presence of a second multi-wave point and the apparent rolled-up vortex instabilities in the combustion product along each slip line extending downstream from the multi-wave points near the initiation region.

Author Contributions: Conceptualization, H.H.T. and H.D.N.; methodology, C.Y. and X.C.M.; Investigation, C.Y., H.H.T. and H.D.N.; writing—original draft preparation, H.D.N.; writing—review and editing, X.C.M. and H.D.N.

Funding: This research is supported by the National Natural Science Foundation of China NSFC (Nos. 11822202 and 91641130) and the Natural Sciences and Engineering Research Council of Canada NSERC (No. RGPIN-2017-06698).

Conflicts of Interest: The authors declare no conflict of interest.

\section{References}

1. Urzay, J. Supersonic combustion in air-breathing propulsion systems for hypersonic flights. Annu. Rev. Fluid Mech. 2018, 50, 593-627. [CrossRef]

2. Heiser, W. Hypersonic Airbreathing Propulsion; AIAA Education Series; AIAA: Washington, DC, USA, 1994.

3. Kailasanath, K. Review of propulsion applications of detonation waves. AIAA J. 2000, 38, 1698-1708. [CrossRef]

4. Wolanski, P. Detonative propulsion. Proc. Combust. Inst. 2013, 34, 125-158. [CrossRef]

5. Roy, G.D.; Frolov, S.M.; Borisov, A.A.; Netzer, D.W. Pulse detonation propulsion: Challenges, current status, and future perspective. Prog. Energy Combust. Sci. 2004, 30, 545-672. [CrossRef]

6. Hertzberg, A.; Bruckner, A.P.; Knowlen, C. Experimental investigation of ram accelerator propulsion modes. Shock Waves 1991, 1, 17-25. [CrossRef]

7. Higgins, A.J. Ram accelerators: Outstanding issues and new directions. J. Propul. Power 2006, 22, $1170-1187$. [CrossRef]

8. Chan, J.; Sislian, J.P.; Alexander, D. Numerically simulated comparative performance of a Scramjet and Shcramjet at Mach 11. J. Propul. Power 2010, 26, 1125-1134. [CrossRef]

9. Alexander, D.C.; Sislian, J.P. A computational study of the propulsive characteristics of a Shcramjet engine. J. Propul. Power 2008, 24, 34-44. [CrossRef]

10. Behrens, H.; Struth, W.; Wecken, F. Studies of hypervelocity firings into mixtures of hydrogen with air or with oxygen. Symp. (Int.) Combust. 1965, 10, 245-252. [CrossRef]

11. McVey, J.B.; Toong, T.Y. Mechanism of instabilities of exothermic hypersonic blunt-body flows. Combust. Sci. Technol. 1971, 3, 63-76. [CrossRef]

12. Ju, Y.; Masuya, G.; Sasoh, A. Numerical and theoretical studies on detonation initiation by a supersonic projectile. Symp. (Int.) Combust. 1998, 27, 2225-2231. [CrossRef]

13. Ju, Y.; Sasoh, A.; Masuya, G. On the Detonation Initiation by a Supersonic Sphere. In Ram Accelerators; Takayama, K., Sasoh, A., Eds.; Springer: Berlin/Heidelberg, Germany, 1998.

14. Vasiljev, A.A. Initiation of gaseous detonation by a high speed body. Shock Waves 1994, 3, 21-326. [CrossRef]

15. Lee, J.H.S. Initiation of detonation by a hypervelocity projectile. Prog. Astronaut. Aeronaut. 1997, 173, $293-310$.

16. Kasahara, J.; Fujiwara, T.; Endo, T. Chapman-Jouguet oblique detonation structure around hypersonic projectiles. AIAA J. 2001, 39, 1553-1561. [CrossRef]

17. Maeda, S.; Kasahara, J.; Matsuo, A. Oblique detonation wave stability around a spherical projectile by a high time resolution optical observation. Combust. Flame 2012, 159, 887-896. [CrossRef]

18. Kaneshige, M.J.; Shepherd, J.E. Oblique detonation stabilized on a hypervelocity projectile. Symp. (Int.) Combust. 1996, 26, 3015-3022. [CrossRef]

19. Verreault, J.; Higgins, A.J. Initiation of detonation by conical projectiles. Proc. Combust. Inst. 2011, 33, $2311-2318$. [CrossRef] 
20. Morris, C.I.; Kamel, M.R.; Hanson, R.K. Shock-induced combustion in high-speed wedge flows. Symp. (Int.) Combust. 1998, 27, 2157-2164. [CrossRef]

21. Viguier, C.; Da Silva, L.F.F.; Desbordes, D.; Deshaies, B. Onset of oblique detonation waves: Comparison between experimental and numerical results for hydrogen-air mixtures. Symp. (Int.) Combust. 1996, 26, 3023-3031. [CrossRef]

22. Lu, F.K.; Fan, H.; Wilson, D.R. Detonation waves induced by a confined wedge. Aerosp. Sci. Technol. 2006, 10, 679-685. [CrossRef]

23. Cambier, J.L.; Adelman, H.; Menees, G.P. Numerical simulations of an oblique detonation wave engine. J. Propul. 1990, 6, 315-323. [CrossRef]

24. Ostrander, M.; Hyde, J.; Young, M.; Kissinger, R.; Pratt, D. Standing oblique detonation wave engine performance. In Proceedings of the 23rd Joint Propulsion Conference, San Diego, CA, USA, 29 June-2 July 1987. No. AIAA-87-2002.

25. Adelman, H.G.; Cambier, J.L.; Menees, G.P.; Balboni, J.A. Analytical and experimental validation of the oblique detonation wave engine concept. In Proceedings of the 26th Aerospace Sciences Meeting, Reno, NV, USA, 11-14 January 1988. No. AIAA-88-0097.

26. Gross, R.A. Oblique detonation waves. AIAA J. 1963, 1, 1225-1227. [CrossRef]

27. Pratt, D.T.; Humphrey, J.W.; Glenn, D.E. Morphology of standing oblique detonation waves. J. Propul. Power 1991, 7, 837-845. [CrossRef]

28. Ashford, S.A.; Emanuel, G. Wave angle for oblique detonation waves. Shock Waves 1994, 3, $327-329$. [CrossRef]

29. Emanuel, G.; Tuckness, D.G. Steady, oblique, detonation waves. Shock Waves 2004, 13, 445-451. [CrossRef]

30. Li, C.; Kailasanath, K.; Oran, E.S. Detonation structure behind oblique shocks. Phys. Fluids 1994, 6, 1600-1611. [CrossRef]

31. Lee, J.H.S. Detonation Phenomenon; Cambridge University Press: New York, NY, USA, 2008.

32. Betelin, V.B.; Nikitin, V.F.; Smirnov, N.N.; Smirnova, M.N.; Stamov, L.I.; Tyurenkova, V.V. Using GPUs for solving problems of combustion and physicochemical transformations. Math. Models Comput. Simul. 2017, 9, 727-741. [CrossRef]

33. Vanka, S.P. Computational fluid dynamics on graphics processing units. J. Fluids Eng. 2013, $135,061401$. [CrossRef]

34. Smirnov, N.N.; Nikitin, V.F.; Stamov, L.I.; Nerchenko, V.A.; Tyrenkova, V.V. Numerical simulations of gaseous detonation propagation using different supercomputing architectures. Int. J. Comput. Meth. 2017, 14, 1750038. [CrossRef]

35. Papalexandris, M.V. A numerical study of wedge-induced detonations. Combust. Flame 2000, 120, 526-538. [CrossRef]

36. Teng, H.H.; Jiang, Z.L. On the transition pattern of the oblique detonation structure. J. Fluid Mech. 2012, 713, 659-669. [CrossRef]

37. Liu, Y.; Liu, Y.S.; Wu, D.; Wang, J.P. Structure of an oblique detonation wave induced by a wedge. Shock Waves 2016, 26, 161-168. [CrossRef]

38. Wang, T.; Zhang, Y.; Teng, H.H.; Jiang, Z.L.; Ng, H.D. Numerical study of oblique detonation wave initiation in a stoichiometric hydrogen-air mixture. Phys. Fluids 2015, 27, 096101. [CrossRef]

39. Teng, H.H.; Ng, H.D.; Jiang, Z.L. Initiation characteristics of wedge-induced oblique detonation wave in a stoichiometric hydrogen-air mixture. Proc. Combust. Inst. 2017, 36, 2735-2742. [CrossRef]

40. Ren, Z.; Wang, B.; Xiang, G.; Zhao, D.; Zheng, L. Supersonic spray combustion subject to scramjets: Progress and challenges. Prog. Aerosp. Sci. 2019, 105, 40-59. [CrossRef]

41. Iwata, K.; Nakaya, S.; Tsue, M. Wedge-stabilized oblique detonation in an inhomogeneous hydrogen-air mixture. Proc. Combust. Inst. 2017, 36, 2761-2769. [CrossRef]

42. Yu, M.; Miao, S. Initiation characteristics of wedge-induced oblique detonation waves in turbulence flows. Acta Astronaut. 2018, 147, 195-204. [CrossRef]

43. Zhang, Y.; Teng, H.H.; Ng, H.D.; Wen, C. Transition between different initiation structures of wedge-induced oblique detonations. AIAA J. 2018, 56, 4016-4023. [CrossRef]

44. Zhang, Y.N.; Gong, J.S.; Wang, T. Numerical study on initiation of oblique detonations in hydrogen-air mixtures with various equivalence ratios. Aerosp. Sci. Technol. 2016, 49, 130-134. [CrossRef] 
45. Liu, Y.; Wang, L.; Xiao, B.; Yan, Z.; Wang, C. Hysteresis phenomenon of the oblique detonation wave. Combust. Flame 2018, 192, 170-179. [CrossRef]

46. Vlasenko, V.V.; Sabelnikov, V.A. Numerical simulation of inviscid flows with hydrogen combustion behind shock waves and in detonation waves. Combust. Explos. Shock Waves 1995, 31, 376-389. [CrossRef]

47. Da Silva, L.F.F.; Desbordes, D.; Deshaies, B. Stabilization of an oblique detonation wave by a wedge: A parametric numerical study. Combust. Flame 2000, 121, 152-166. [CrossRef]

48. Ng, H.D.; Radulescu, M.I.; Higgins, A.J.; Nikiforakis, N.; Lee, J.H.S. Numerical investigation of the instability for one-dimensional Chapman-Jouguet detonations with chain-branching kinetics. Combust. Theory Modell. 2005, 9, 385-401. [CrossRef]

49. Yang, P.F.; Teng, H.H.; Jiang, Z.L.; Ng, H.D. Effects of inflow Mach number on oblique detonation initiation with a two-step induction-reaction kinetic model. Combust. Flame 2018, 193, 246-256. [CrossRef]

50. Tang-Yuk, K.C.; Mi, X.C.; Lee, J.H.S.; Ng, H.D. Transmission of a detonation across a density interface. Shock Waves 2018, 28, 967-979. [CrossRef]

51. Li, J.; Ning, J. Experimental and numerical studies on detonation reflections over cylindrical convex surfaces. Combust. Flame 2018, 198, 130-145. [CrossRef]

52. Dounia, O.; Vermorel, O.; Misdariis, A.; Poinsot, T. Influence of kinetics on DDT simulations. Combust. Flame 2019, 200, 1-14. [CrossRef]

53. Smirnov, N.N.; Penyazkov, O.G.; Sevrouk, K.L.; Nikitin, V.F.; Stamov, L.I.; Tyurenkova, V.V. Onset of detonation in hydrogen-air mixtures due to shock wave reflection inside a combustion chamber. Acta Astronaut. 2018, 149, 77-92. [CrossRef]

54. Taylor, B.; Kessler, D.; Gamezo, V.; Oran, E.S. Numerical simulations of hydrogen detonations with detailed chemical kinetics. Proc. Combust. Inst. 2013, 34, 2009-2016. [CrossRef]

55. Powers, J.M. Review of multiscale modeling of detonation. J. Propul. Power 2006, 22, 1217-1229. [CrossRef]

56. Zhang, Y.; Fang, Y.; Ng, H.D.; Teng, H.H. Numerical investigation on the initiation of oblique detonation waves in stoichiometric acetylene-oxygen mixtures with high argon dilution. Combust. Flame 2019, 204, 391-396. [CrossRef]

57. Radulescu, M.I.; Sharpe, G.J.; Bradley, D. A universal parameter quantifying explosion hazards, detonability and hot spot formation: $\chi$ number. In Proceedings of the Seventh International Seminar Fire \& Explosion Hazards, Providence, RI, USA, 5-10 May 2013.

58. Tang, J.; Radulescu, M.I. Dynamics of shock induced ignition in Fickett's model: Influence of $\chi$. Proc. Combust. Inst. 2013, 34, 2035-2041. [CrossRef]

59. Li, C.; Kailasanath, K.; Oran, E.S. Effects of boundary layers on oblique detonation structures. In Proceedings of the 31st Aerospace Sciences Meeting \& Exhibit, Reno, NV, USA, 11-14 January 1993. No. AIAA-93-0450.

60. Bourlioux, A. Numerical Study of Unstable Detonations. Ph.D. Thesis, Princeton University, Princeton, NJ, USA, 1991.

61. Higgins, A.J. Steady One-Dimensional Detonations. In Shock Wave Science and Technology Library; Zhang, F., Ed.; Springer: Berlin, Germany, 2012; Volume 6, Chap. 2.

62. Teng, H.H.; Jiang, Z.L.; Ng, H.D. Numerical study on unstable surfaces of oblique detonations. J. Fluid Mech. 2014, 744, 111-128. [CrossRef]

63. Verreault, J.; Higgins, A.J.; Stowe, R.A. Formation of transverse waves in oblique detonations. Proc. Combust. Inst. 2013, 34, 1913-1920. [CrossRef]

64. Zhang, Y.; Zhou, L.; Gong, J.; Deng, X.; Ng, H.D.; Teng, H.H. Effects of activation energy on the instability of oblique detonation surfaces with a one-step chemistry model. Phys. Fluids 2018, 30, 106110.

65. Teng, H.H.; Ng, H.D.; Li, K.; Luo, C.T.; Jiang, Z.L. Evolution of cellular structure on oblique detonation surfaces. Combust. Flame 2015, 162, 470-477. [CrossRef]

66. Grismer, M.J.; Powers, J.M. Numerical predictions of oblique detonation stability boundaries. Shock Waves 1996, 6, 147-156. [CrossRef]

67. Toro, E.F. Riemann Solvers and Numerical Methods for Fluid Dynamics, 3rd ed.; Springer: Berlin, Germany, 2009.

68. Kiyanda, C.B.; Morgan, G.H.; Nikiforakis, N.; Ng, H.D. High resolution GPU-based flow simulation of the gaseous methane-oxygen detonation structure. J. Vis. 2015, 18, 273-276. [CrossRef]

69. Mi, X.C.; Higgins, A.J.; Ng, H.D.; Kiyanda, C.B.; Nikiforakis, N. Propagation of gaseous detonation waves in a spatially inhomogeneous reactive medium. Phys. Rev. Fluids 2017, 2, 053201. [CrossRef] 
70. Mi, X.C.; Higgins, A.J.; Kiyanda, C.B.; Ng, H.D.; Nikiforakis, N. Effect of spatial inhomogeneities on detonation propagation with yielding confinement. Shock Waves 2018, 28, 993-1009. [CrossRef]

71. Smirnov, N.N.; Betelin, V.B.; Nikitin, V.F.; Stamov, L.I.; Altoukhov, D.I. Accumulation of errors in numerical simulations of chemically reacting gas dynamics. Acta Astronaut. 2015, 117, 338-355. [CrossRef]

72. Smirnov, N.N.; Betelin, V.B.; Shagaliev, R.M.; Nikitin, V.F.; Belyakov, I.M.; Deryuguin, Y.N.; Aksenov, S.V.; Korchazhkin, D.A. Hydrogen fuel rocket engines simulation using LOGOS code. Int. J. Hydrogen Energy 2014, 39, 10748-10756. [CrossRef]

73. Ng, H.D.; Zhang, F. Detonation Instability. In Shock Wave Science and Technology Library; Zhang, F., Ed.; Springer: Berlin, Germany, 2012; Volume 6, Chap. 3.

74. Gamezo, V.N.; Desbordes, D.; Oran, E.S. Formation and evolution of two-dimensional cellular detonations. Combust. Flame 1999, 116, 154-165. [CrossRef]

75. Choi, J.Y.; Ma, F.; Yang, V. Some numerical issues on simulation of detonation cell structures. Combust. Explos. Shock Waves 2008, 44, 560-578. [CrossRef]

76. Choi, J.Y.; Kim, D.W.; Jeung, I.S.; Ma, F.; Yang, V. Cell-like structure of unstable oblique detonation wave from high-resolution numerical simulation. Proc. Combust. Inst. 2007, 31, 2473-2480. [CrossRef]

77. Teng, H.H.; Morgan, G.H.; Kiyanda, C.B.; Nikiforakis, N.; Ng, H.D. GPU-based simulation of the two-dimensional unstable structure of gaseous oblique detonations. AIP Conf. Proc. 2015, 1648, 030027.

78. Gui, M.Y.; Fan, B.C. Wavelet structure of wedge-induced oblique detonation waves. Combust. Sci. Technol. 2012, 184, 1456-1470. [CrossRef]

79. Mahmoudi, Y.; Karimi, R.; Deiterding, R.; Emami, S. Hydrodynamic instabilities in gaseous detonations: Comparison of Euler, Navier-Stokes, and large-eddy simulation. J. Propul. Power 2014, 30, 384-396. [CrossRef]

80. Ziegler, J.L.; Deiterding, R.; Shepherd, J.E.; Pullin, D.I. An adaptive high-order hybrid scheme for compressive, viscous flows with detailed chemistry. J. Comput. Phys. 2011, 230, 7598-7630. [CrossRef]

(C) 2019 by the authors. Licensee MDPI, Basel, Switzerland. This article is an open access article distributed under the terms and conditions of the Creative Commons Attribution (CC BY) license (http://creativecommons.org/licenses/by/4.0/). 